\title{
Molecular Characterization of Goats Using Random Amplified Polymorphic Dna
}

\author{
Nandani Kumari, L.B. Singh and Subodh Kumar \\ Departmant of Animal Breeding and Genetics, \\ Ranchi Veterinary College, Birsa Agricultural University Jharkhand, 835240, India
}

Received 2011-10-01, Revised 2012-10-30; Accepted 2013-05-10

\begin{abstract}
Black Bengal goat is a very valuable goat breed in India. The present investigation was proposed to be undertaken to evaluate gene flow, phylogeographic history, genetic structure and differentiation of both the goat populations to study genetic variation prevailing among Black Bengal and Black Bengal type goat in west Bengal and Jharkhand respectively and to study genetic relationship between both goat populations and to study population structure and gene flow between these two goat populations. This experiment was conducted in the department of animal breeding and genetics (molecular biology laboratory). DNA was extracted from 50 goats from each of the Black Bengal and Black Bengal type goats of Jharkhand and the extracted DNA was observed by gel electrophoresis. Ten specific goat specific primers were obtained from sigma. All the primers except for primers one and two were capable of priming polymorphic amplification pattern in both the breeds. Random Amplification of Polymorphic DNA-Polymerase Chain Reaction (RAPD-PCR) analysis was carried out using DNA samples of 50 Black Bengal goat and 50 Black Bengal type goats of Jharkhand (referred to as Jharkhand black) goat breeds. Only unambiguous, reproducible and scorable polymorphic fragments were taken into consideration for analysis. The amplification pattern of representative samples of Black Bengal showed that primers 6,7,8,9 were highly polymorphic. Polymorphism Information Content (PIC) or expected heterozygosity scores varied from a minimum of 0.219 for primer sigma 06-3 to a maximum of 0.486 for primer sigma 04 with over all mean 0.411 in Black Bengal goat. Similarly for Jharkhand Black, the value of PIC ranged from a minimum of 0.278 for primer sigma 06-3-0.496 for primer sigma10-2 with over all mean value of 0.413 . Data were analyzed by using a computer programe POPGENE (Version 1.31). A more appropriate measure of genetic variation within a population is gene diversity .It was observed that, mean value of Nei,s Gene diversity value (h) was 0.3750 for Black Bengal and was 0.4022 for Jharkhand black. In case of Black Bengal, it was found that the maximum value of Nei's gene diversity was 0.4861 for locus sigma 6-2 and sigma 9-2 while the minimum value of 0.0664 was found for locus Sigma 04. In Jharkhand Black the maximum value of Nei's gene diversity $(\mathrm{h})$ was found to be 0.4965 for locus Sigma 10-2 and sigma06-1and the minimum value was 0.2491 for locus sigma 08-1. Dendrogram based on Nei's genetic distance using Unweighted Pair Group Method of Arithmetic Means (UPGMA) indicated relative closeness and distance of the two goat breed. This study was mainly done on breeds raised in marginal agricultural areas in order to assist in situ conservation. The result was important with respect to puzzle of goat genetic diversity and conservation. Therefore it can be recommended that within breed diversity is actively maintained to enable these extensively unmanaged stocks to adapt to future demands and conditions and there is ample scope for further improvement in its productivity through appropriate breeding strategies.
\end{abstract}

Keywords: Black Bengal, Molecular Characterization, Genetic Distance, Situ Conservation

Corresponding Author: Nandani Kumari, Department of Animal Breeding and Genetics, Ranchi Veterinary College, Birsa Agricultural University Jharkhand, 835240, India Tel: -09835396685 


\section{INTRODUCTION}

Goat is the earliest ruminant domesticated around 9000-7000 B.C. Goat is a hollow-horned ruminant belonging to the mammalian order Artiodactyla, suborder ruminantia, family bovidae and either of genera Capra or Hemitragus. Goat belongs to the genus capra and has been developed from following five wild species (i) Capra hircus including Bezoar (ii) Capra ibex (iii) Capra caucasia the Caucasian fur (iv) Capra pyrenaican and (v) Capra falconeri, the markhor. Black Bengal goat is believed to be derived from wild Bezoar of Capra aegragrus (Herre and Rohrs, 1973) with infiltrated blood from Markhor i.e., Capra falconeri. In nature, genetic erosion and degradation is a continuous process. The agro biological resources are declining very fast. Worldwide there are 4,500 breeds, of which more than $30 \%$ are at the risk of loss. In India, $50 \%$ of indigenous goats, $30 \%$ of indigenous sheep and $20 \%$ of indigenous cattle and almost all poultry breeds are threatened (FAO, 1997). India possesses the richest source of germplasm of goats. There are 20 well known distinct breeds in India. Black Bengal is one of the breeds of goats in India. On this note, it is essential to mention that, both the goat populations viz. Black Bengal and Black Bengal type goats of Jharkhand are black in color but differ in morphological characters such as body weight and these two goat populations also show significant divergence to each other in molecular study based on Microsatellite marker (Kumar, 2007). The classification of these breeds based on phenotypic and/or geographical distribution may not actually characterize the genetic structure of the populations because genetically similar individual might be labeled differently due to distinct geography and or phenotype. Moreover, they share their breeding tract and thereby there may be intermixing of these population/breeds which may cause dilution of genetic structure, if exists. Estimation of genetic variation are increasingly being based upon information at the DNA level by various molecular techniques such as Randomly amplified polymorphic DNA, (RAPD), Amplified Fragment Length Polymorphism (AFLP), RFLP, SSR and microsatellite. Among them, RAPD, markers generated by Polymerase Chain Reaction (PCR) is widely used since 1990's to assess intra specific genetic variation at nuclear level (Welsh and McClellan, 1990). RAPD is a PCR based technique for identifying genetic variation. It involves use of single arbitrary primer in a PCR reaction, resulting in amplification of many discrete DNA. RAPD technology provides a quick and efficient screen for DNA sequence based polymorphism at a very large number of loci. The major advantage of RAPD includes that, it does not require pre-sequencing of DNA. The vast range of potential primers that can be used, give the technique great diagnostic power. Reproducible RAPD bands can be found by careful selection of primers, optimization of PCR condition for target species and replication to ensure that only reproducible bands are scored. RAPD analysis has been extensively used for various purposes which include identification and classification of accessions (Fukuoka et al., 1992; Virk et al., 1995), identification of breeds (Qian et al., 1996) and genetic diversity analysis (Yu and Paul, 1992; Mackill, 1995; Cao and Oard, 1997).

\section{MATERIALS AND METHODS}

Step 1: Fifty blood samples were collected each from Black Bengal goat and Jharkhand Black goat .Genomic DNA was isolated and purified from white blood cells using Proteinase $\mathrm{K}$ digestion and standard phenol: chloroform extraction method as per the standard protocol described by Sambrook et al. (1989).

Step 2: Agarose gel electrophoresis for checking the quality of isolated genomic DNA samples was done.

Step 3: This was followed by Polymerase chain reaction and PCR technique was used to amplify the chosen marker. For this, optimization of PCR technique was followed by PCR of DNA samples with ten random primers (Table 1).

Step 4: Agarose gel electrophoresis of PCR amplified product of DNA was done and the bands were obtained (Fig. 1).

Step 7: The amplified product were statistically analyzed to measure the between and within population diversity.

Step 8: The genetic diversity within and between population was analyzed as the observed and expected number of alleles and Shannon's information Index using popgene software.

Step 9: Ewan's Watterson test was performed to test the neutrality for RAPD markers; the statistics $\mathrm{F}$ (sum of square of allelic frequency) and limit (upper and lower) at 95\% confidence region for the test were calculated using the algorithm by mainly using 100 simulated samples and implemented in popgene software package. 


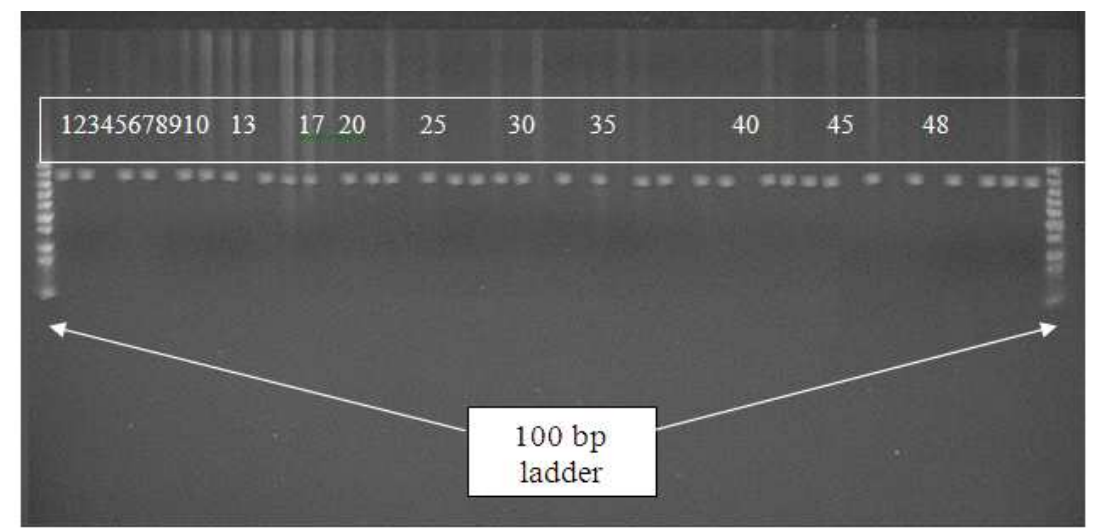

Fig. 1. Jharkhand black goat genomic (48 samples) amplification with primer sigma03

Table 1. Details of random primers

\begin{tabular}{lllll}
\hline Oligo name & Oligo sequence & $\begin{array}{l}\text { Length } \\
\text { (bases) }\end{array}$ & Tm & GC\% \\
\hline SIGMA01 & GTCCCGAAAC & 10 & 32.7 & 60 \\
SIGMA02 & ACAGTCGCAG & 10 & 29.2 & 50 \\
SIGMA03 & AGGCTTAAGC & 10 & 28.9 & 50 \\
SIGMA04 & CCACTACCCA & 10 & 29.4 & 60 \\
SIGMA05 & GCAGCTGTGC & 10 & 35.0 & 70 \\
SIGMA06 & GGACCTGTGC & 10 & 32.9 & 70 \\
SIGMA07 & ATAACCAGCC & 10 & 28.2 & 50 \\
SIGMA08 & ACTGCAGGCT & 10 & 33.1 & 60 \\
SIGMA09 & ACGGTGTACC & 10 & 27.7 & 60 \\
SIGMA10 & CGCCGCATAT & 10 & 41.7 & 60 \\
\hline
\end{tabular}

Step 10: In order to quantify the percentage of molecular variance due to differences among the difference among different populations and significance was tested by a non-random permutation approach using AMOVA programme included in Arleqin software package. Migration rate which showed the gene flow per generation was also found out. A dendrogram by UPGMA method was constructed.

\section{RESULTS}

\subsection{Black Bengal Goat Genomic (48 Samples) Amplification with Primer Sigma03}

The result can be summarized as follows.

\subsection{Gene Frequency}

Gene frequency in Black Bengal ranged from 0.1250.729 for allele 0 and from $0.271-0.875$ for allele 1 . Similarly in case of Jharkhand Black, Gene frequency ranged from $0.146-0.625$ for allele 0 and from 0.375 0.854 for allele 1 .

\subsection{Polymorphic Information Content}

Polymorphic Information Content (PIC) or expected heterozygosity scores varied from 0.2190.486 with overall mean 0.411 in Black Bengal and from 0.278-0.496 with overall mean 0.413 in Jharkhand Black goat (Table 2).

\subsection{Mean Observed and Effective Number of Alleles}

Mean observed number of alleles was 2 in both Jharkhand Black and Black Bengal with mean effective number of alleles was 1.6991 for Black Bengal and 1.6935 for Jharkhand Black.

\subsection{Nei's Gene Diversity Value (h)}

Nei's gene diversity value was 0.3750 for Black Bengal and 0.4022 for Jharkhand Black.

\subsection{Shannon's Information Index}

Shannon's Information Index was 0.6792 for Black Bengal and 0.5898 for Jharkhand Black.

\subsection{Gene flow}

Gene flow (Nm) value was 25.68, Hs (Mean sample gene diversity) between two population was 0.412 and $\mathrm{Ht}$ (Total gene diversity )in total sample was 0.420 .

\subsection{Genetic Identity and Genetic Distance}

Nei's genetic identity was found to be 0.9727 and genetic distance was 0.0276 . 
Table 2. Polymorphoic information content value

\begin{tabular}{llr}
\hline Allele $\backslash$ locus & Black Bengal & Jharkhand black \\
\hline SIGMA03 & 0.469 & 0.413 \\
SIGMA04 & 0.486 & 0.395 \\
SIGMA05 & 0.469 & 0.395 \\
SIGMA06-1 & 0.395 & 0.496 \\
SIGMA06-2 & 0.413 & 0.375 \\
SIGMA06-3 & 0.219 & 0.278 \\
SIGMA06-4 & 0.478 & 0.469 \\
SIGMA07-1 & 0.395 & 0.353 \\
SIGMA07-2 & 0.469 & 0.429 \\
SIGMA07-3 & 0.429 & 0.478 \\
SIGMA08-1 & 0.375 & 0.249 \\
SIGMA08-2 & 0.375 & 0.469 \\
SIGMA08-3 & 0.375 & 0.413 \\
SIGMA09-1 & 0.457 & 0.375 \\
SIGMA09-2 & 0.329 & 0.395 \\
SIGMA09-3 & 0.353 & 0.457 \\
SIGMA10-1 & 0.478 & 0.491 \\
SIGMA10-2 & 0.429 & 0.496 \\
Mean & 0.411 & 0.413 \\
SD & 0.068 & 0.071 \\
\hline
\end{tabular}

\subsection{Evan's Watterson's Test of Neutrality}

Not even a single locus showed the F value beyond the standardized range of U95 and L95 at 95\% confidence level and so all the locus were neutral to selection pressure when these were taken separately but when combined together the two locus SIGMA06-4 and SIGMA10-1 were not found neutral to selection pressure.

\subsection{Dendrogram}

The clustering between the breeds was not very sharp with their intermingling at a few places. This showed the dilution between the gene pools of Black Bengal and Jharkhand Black.

\subsection{AMOVA}

The Analysis of Molecular Variance (AMOVA) showed $1.69983 \%$ among population variation and $98.30017 \%$ between population variation.

\section{DISCUSSION}

A significant amount of differentiation among the twobreeds and high level of gene flow between Jharkhandblack and Black Bengal was observed. As Jharkhand black Goat also shows significant gene diversity, it should be given a separate identity.
The result was crucial for in situ conservation and on the basis of this result, it can be recommended that within breed diversity is actively maintained to enable these extensively unmanaged stockiest to adapt to further demands and conditions and there is amplescope for further improvement in its productively through appropriate breeding strategies. The results presented added important information on the puzzle of goat genetic diversity and conservation in India where it is of crucial economic relevance to increasingly marginalized rural communities. Therefore, it can be recommended that within-breed diversity is actively maintained to enable these extensively unmanaged stocks to adapt to future demands and conditions and there is ample scope for further improvement in its productivity through appropriate breeding strategies.

\section{CONCLUSION}

On a whole it was concluded that Jharkhand black shows considerable amount of similarities and few of dissimilarities and so Jharkhand black may be a strain or the derivative of Black Bengal and should be given a separate identity considering the importance of gene conservation amidst high gene flow due to geographical closeness.

\section{REFERENCES}

Cao, D. and J.H. Oard, 1997. Pedigree and RAPD-based DNA analysis of commercial U.S. rice cultivars. Crop Sci., 37: 1630-1635.

FAO, 1997. The global strategy for the management of farm animal genetic resources: A call for action. FAO Publishing, Rome.

Fukuoka, S., K. Hosaka and O. Kamijima, 1992. Use of Random Amplified Polymorphic DNAs (RAPDs) for identification of rice accessions. Jap. J. Genet., 67: 243-252. DOI: 10.1266/jjg.67.243

Herre, W. and M. Rohrs, 1973. Haustiere: Zoologisch Gesehen. Gustav Fischer Verlag, Stuttgart, ISBN-10: 3437301497, pp: 240.

Kumar, S., 2007. Molecular characterization of some goat genetic resources. Birsa Agricultural University, Ranchi.

Mackill, D.J., 1995. Classifying japonica rice cultivars with RAPD markers. Crop Sci., 35: 889-894.

Qian, Q., C. Hong, S. Zongxiu and Z. Lihuang, 1996. The study on determining true and false hybrid rice II you 63 using RAPD molecular markers. Chin. J. Rice Sci., 10: 241-242. 
Sambrook, J., E.F. Fritsch and T. Maniatis, 1989. Molecular Cloning: A Laboratory Manual. 2nd Edn., Cold Spring Harbor Laboratory Press, ISBN-10: 0879693096.

Virk, P.S., H.J. Newbury, M.T. Jackson and B.V. FordLloyd, 1995. The identification of duplicate accessions within a rice germplasm collection using RAPD analysis. Theory Applied Genet., 90: 10491055. DOI: $10.1007 / \mathrm{BF} 00222920$
Welsh, J. and M. McClellan, 1990. Fingerprinting genomes using PCR with arbitrary primers. Nucl. Acids Res., 18: 7213-7218. PMID: 2259619

Yu, K.F. and K.P. Pauls, 1992. Optimization of the PCR program for RAPD analysis. Nucl. Acid Res., 20: 2606-2606. DOI: 10.1093/nar/20.10.2606 